\title{
Hypermethylation-mediated Transcriptional Silencing of IncRNA-SCARF1 Promotes Progression and Metastasis of Hepatocellular Carcinoma
}

\section{Boyi Liao}

Fudan University, Fudan University

\section{Peiran Huang}

Fudan University, Fudan University

Xiangyu Zhang

Fudan University, Fudan University

\section{Xinyu Wang}

Fudan University, Fudan University

\section{Kaiqian Zhou}

Fudan University, Fudan University

\section{Feiyu Chen}

Fudan University, Fudan University

Cheng Zhou

Fudan University, Fudan University

Lei Yu

Fudan University, Fudan University

Jie Hu

Fudan University, Fudan University

\section{Zheng Wang}

Fudan University, Fudan University

Jian Zhou ( $\nabla$ zhou.jian@zs-hospital.sh.cn )

Fudan University, Fudan University

\section{Research Article}

Keywords: Hepatocellular carcinoma, LncRNA, Microarray, CUL9

Posted Date: December 30th, 2021

DOI: https://doi.org/10.21203/rs.3.rs-1110965/v1

License: (c) (i) This work is licensed under a Creative Commons Attribution 4.0 International License. Read Full License 
Page $2 / 22$ 


\section{Abstract}

Background: Hepatocellular carcinoma (HCC) is one of the most common causes of cancer-related deaths. Recent studies have demonstrated that deregulation of long noncoding RNAs (IncRNAs), such as abnormal DNA methylation of promoter, is strongly associated with development and progression of diverse malignant tumors. This study investigated the mechanisms and changes in DNA methylation levels of promoter regions of HCC-specific IncRNAs, and alterations of downstream target genes.

Methods: LncRNA expression profile data of 8 human HCC tissues and matched normal tissues were obtained. LncRNAs with aberrant methylation were identified through DNA methylation microarray. The biological functions of the IncRNAs were investigated through targeted knockdown of IncRNA-SCARF1 in vitro and in vivo. Furthermore, the downstream targets of IncRNA-SCARF1 were identified through ChIRPMS.

Results: LncRNA-SCARF1 was significantly down-regulated in HCC samples. Hypermethylation in the promoter of Inc-SCARF1 induced its down-regulation in HCC. Over-expression of Inc-SCARF1 inhibited the tumor proliferation and migration ability of HCC cells in vitro and in vivo. Furthermore, CUL9 was found to be a potential downstream target of IncRNA-SCARF1.

Conclusion: LncRNA-SCARF1 regulates HCC progression by interacting with CUL9 and may serve as a prognostic biomarker or an effective therapeutic target in HCC.

\section{Background}

Primary liver cancer, which accounts for over 840,000 new cases per year, is the third leading cause of cancer-related deaths worldwide(1). Hepatocellular carcinoma (HCC), which is characterized by obscure symptoms and a propensity for hematogenous metastasis, is the most frequently occurring pathological type, accounting for about $90 \%$ of all cases. Although great progress has been made in development of multiple therapeutic modalities, such as surgical options, non-ablative treatment and targeted therapy(2), prognosis of HCC patients remains poor due to tumor heterogeneity and high malignancy(3). Previous studies have associated occurrence of HCC with various factors, including virus infection, alcohol abuse and $\mathrm{NASH}(4)$. For decades, activation of oncogenes as well as inactivation and mutation of suppressor genes have been the most popular predictors of HCC carcinogenesis $(5,6)$. To date, however, the specific molecular mechanism underlying $\mathrm{HCC}$ occurrence and progression remains elusive despite numerous researches on the subject.

Previous studies have demonstrated that the human genome encodes a spot of protein-coding genes, only representing $<2 \%$ of the total genome sequence(7). However, more than $90 \%$ of the noncoding RNAs (ncRNAs) are actively transcribed. Long noncoding RNAs (IncRNAs), a class of ncRNAs with a length of at least 200 nucleotides that are extensively expressed during genome transcription ", have been shown to participate in specific biological processes(8). Therefore, they are considered a generalist due to their involvement in each step of gene regulation. Recent studies have demonstrated that dysregulation of 
IncRNAs, such as abnormal DNA methylation of promotor(9) and loss of genomic imprinting(10), is strongly associated with development and progression of diverse human diseases, including prostate and nasopharyngeal cancers $(11,12)$. Additional evidences have revealed that some IncRNAs, such as SNHG7 and PDPK2P, can promote progression of HCC and are closely associated with poor prognosis $(13,14)$.

Apart from their regulatory functions on cellular physiology, aberrant expression of IncRNAs via epigenetic regulation has also attracted numerous research attention. Previous studies have indicated that DNA methylation of $\mathrm{CpG}$ islands of the promoter regions regulate down-regulation of the tumor suppressor genes(15). Similarly, abnormal DNA methylation of IncRNAs promoters has emerged as a driver for malignancies(16). Although previous studies have shown that DNA methylation is a predictor for poor survival of HCC patients(17), interaction between DNA methylation and expression patterns of HCC-related IncRNAs remains unknown. In the present study, we investigated the mechanisms underlying regulation of HCC-specific IncRNAs coupled with the changes in DNA methylation levels in promoter regions and the alterations associated with downstream target genes. Our findings indicate that IncRNAs are a novel molecular marker and therapeutic target for HCC.

\section{Methods}

\section{Patient recruitment and tissue collection}

We recruited $8 \mathrm{HBV}$-related and pathologically confirmed primary HCC patients, who received radical hepatic resection at the Zhongshan hospital, Fudan university between October and December in 2011. None of the patients had received any auxiliary therapy before surgery. Primary HCC and adjacent normal tissues were obtained from patientsThe study protocol was approved by the Ethics Committee of Zhongshan Hospital, and informed consent was obtained from all subjects prior to inclusion in the study.

\section{Microarray analysis}

Expression profiles of LncRNAs from 8 human HCC and matched normal tissues were analyzed using ArrayStar Human LncRNA Microarray ( $8 \times 60$ k, ArrayStar, Rockville, MD, version 2.0). This dataset comprises a total of 33,045 IncRNAs collected from several databases, such as RefSeq, the UCSC Known genes, and Ensembl. Total RNA was extracted, amplified, and transcribed into fluorescent complementary RNA (cRNA) using the Quick Amp Labeling Kit, One-Color (Palo Alto, CA, USA) according to the manufacturer's instructions. Labeled cRNAs were hybridized onto the human IncRNA arrays, and after washing steps, the arrays were scanned using the Agilent Microarray Scanner (G2565BA) and analyzed with the Feature Extraction software (version 11.0.1.1). Quantile normalization and data processing were performed using the GeneSpring GX software (version 11.5.1, Agilent Technologies). The threshold for screening of differential IncRNAs comprised a fold change $>2.0$ and $P$ value $<0.05$.

Subsequent processing of DNA methylation microarray data was conducted using ArrayStar Human 2.1M LncRNA Promoter Microarray (Rockville, MD, USA). Briefly, total DNA was extracted using the QIAamp DNA 
Mini Kit (QIAGEN, Valencia, CA, USA), sonicated, denatured and immunoprecipitated with anti5- methylcytosine antibodies. The DNA-antibody complex was isolated and purified by immunomagnetic beads (Millipore, Bedford, MA, USA), followed by methylated DNA Immunoprecipitation (MeDIP) as previously described (18). After quality control, MeDIP and input DNA fragments were amplified using the Sigma WGA kit, and purified using the QIAquick PCR purification kit (QIAGEN, Valencia, CA, USA). Next, the fluorescent-labeled MeDIP (Cy5) was mixed with input DNA (Cy3), denatured and hybridized onto the DNA methylation microarray. The mixture was washed, and the arrays scanned and analyzed. To accurately quantify methylation levels of CpGs, we applied a novel analytical methodology, known as modeling experimental data with MeDIP enrichment (MEDME), which utilizes the absolute methylation score (AMS) as an indicator of DNA methylation to identify differentially methylated regions (DMRs) of IncRNA promoters. Next, we merged differentially methylated probes, based on AMS, into candidate DMRs, then recalculated and re-tested the average AMS of candidate DMRs via t-test. Finally, we selected DMRs with average AMS that were significantly different between two groups, and defined them as AMS DMRs.

\section{RNA isolation, reverse transcription and quantitative real time polymerase chain reaction (qRT-PCR)}

Total RNA was isolated from tissues or cells using the TRIzol reagent (Invitrogen), then $2 \mu \mathrm{g}$ of the RNA reverse transcribed to complementary DNA (cDNA) using the High Capacity cDNA Reverse Transcription kit (Ambion Inc., Austin, USA) according to the manufacturer's instructions. qRT-PCR was performed using the Power SYBR Green PCR Master Kit (Applied Biosystems, Foster City, CA) on the ABI 7900HT Fast Real-Time PCR System, targeting specific genes whose primers are listed in Supplementary Table 1. The housekeeping gene glyceraldehyde 3-phosphate dehydrogenase (GADPH) was used as an endogenous control, while relative expression of $L n c R N A s$ was evaluated using the $2^{-\Delta \Delta C t}$ method. All amplifications were performed in triplicates.

\section{Bisulphite modification combining sequencing PCR (BSP) and Methylation specific PCR (MSP)}

Total genomic DNA was isolated from the tissues using the QIAamp DNA Mini Kit (QIAGEN) and $500 \mathrm{ng}$ bisulfate-modified using the Methyl Code ${ }^{\text {TM }}$ Bisulfite Conversion Kit (Invitrogen, Carlsbad, CA, USA). Bisulfate-treated DNA was amplified and cloned into the pMD18-T vector. Methylation level for each site is indicated as the mean percentage of the total methylation on the sequencing data obtained from 10 clones. MSP was conducted as previously described(19), targeting genes whose primers are listed in Supplementary Table 2

\section{Cell cultures}


Human hepatocellular carcinoma cell lines (HepG2, SMMC-7721, MHCC-97L, MHCC-97H, HCC-LM3) and the normal hepatocytes cell line (L02) were obtained from the Liver Cancer Institute, Fudan University (Shanghai, China), while human HCC cell line (Huh-7) was purchased from the Institute of Biochemistry and Cell Biology, Shanghai Institutes for Biological Sciences, Chinese Academy of Sciences (Shanghai, China). Mycoplasma detections revealed negative results in all cell lines. All cells were cultured in Dulbecco's modified eagle medium (DMEM, Gibco-BRL, Grand Island, New York, USA), supplemented with $10 \%$ fetal bovine serum (FBS, Gibco-BRL, Grand Island, New York, USA), and maintained in a humidified incubator with $5 \% \mathrm{CO}_{2}$ and a temperature of $37^{\circ} \mathrm{C}$.

\section{Synthesis of Inc-SCARF1, plasmids and lentivirus construction and transfections}

Full-length Inc-SCARF1(NR_028076) was cloned and synthesized by Sangon (Shanghai, China), and transfected into $\mathrm{pCDH}$ vectors (CD513B-1, bylensystembio science). The resultant pCDH expression constructs were then cloned into Pseudoviral Particles. Thereafter, MHCC-97H or HepG2 cells were cocultured with lentiviral vectors, with non-transfected cells eliminated using puromycin.

\section{Cell proliferation assay}

Proliferation of MHCC-97H and HepG2 cells overexpressing Inc-SCARF1 (Lv-Inc-SCARF1) and controls (LvGFP) was evaluated using the CCK-8 assay kit (Dojindo Laboratories, Kumamoto, Japan), according to the manufacturer's instructions. Briefly, cell lines were seeded into 96-well plates (Corning Costar Corp., Kennebunk, ME, USA), at a density of $3 \times 10^{3}$ per well, and incubated overnight with $200 \mu$ l of cell culture medium at $37^{\circ} \mathrm{C}$. The rate of cell proliferation was determined at 24,48 , and $72 \mathrm{~h}$ after seeding.

\section{Cell invasion assay}

The invasive ability of MHCC97H-Lv-Inc-SCARF1 and HepG2-Lv-Inc-SCARF1 cells were also tested using 6.5-mm Transwells with 8.0- $\mu \mathrm{m}$ pore polycarbonate membrane in 24-well plates (Corning Costar Corp., Kennebunk, ME, USA). Briefly, the transwell chambers were first coated with $100 \mu \mathrm{l}$ Matrigel $(0.8 \mathrm{mg} / \mathrm{ml})$ and incubated for $2 \mathrm{~h}$ at $37^{\circ} \mathrm{C}$. Next, $3 \times 10^{5}$ cells were inoculated into $100 \mu \mathrm{l}$ of serum-free medium, and seeded onto the upper compartment of the chamber, while the lower chamber was loaded with $600 \mu \mathrm{l}$ DMEM supplemented with $10 \%$ FBS, followed by a $24-h$ incubation at $37^{\circ} \mathrm{C}$. The cells and Matrigel on the upper surface were then removed, and invasive cells located on the lower surface fixed and stained with Giemsa. The invasive cells were numbered in five microscopic fields ( $\times 200$ magnification). All individual experiments were performed in triplicate.

\section{Determination of cell apoptosis}


Cell apoptosis was performed using the Annexin V-PE Apoptosis Detection Kit (Becton Dickinson, San Jose, CA, USA) according to the manufacturer's instructions, with apoptotic cells detected via flow cytometry (Becton Dickinson, San Jose, CA, USA).

\section{Western blot assay}

Total proteins were extracted from cell lysates using $100 \mu \mathrm{L}$ pre-cooled lysis buffer $(1 \mathrm{ml}$ RIPA buffer +25 $\mu \mathrm{L}$ PMSF $+110 \mu \mathrm{L}$ Phosphatase inhibitor cocktail), then quantified using the BCA protein quantification kit. Next, $30 \mu \mathrm{g}$ of denatured proteins were separated on a 10\% SDS-PAGE and transferred onto PVDF membranes (Millipore, MA, USA). The membranes were blocked with TBST containing $10 \%$ nonfat milk powder for $2 \mathrm{~h}$, and incubated overnight with primary antibodies of CUL9 at $4^{\circ} \mathrm{C}$. Next, the membranes were washed with TBST for 30 mins and incubated with horseradish peroxidase (HRP)-labeled secondary antibodies (Jackson ImmunoResearch). The membranes were then exposed on the Pierce ECL Plus (Thermo Fisher Scientific, MA, USA) in the ChemiDocTM XRS+ system (Bio-Rad, CA, USA), and images acquired and analyzed using the Image Lab Software.

\section{Chromatin isolation by RNA purification (ChIRP) and mass spectrometry (MS)}

MHCC-97H and HepG2 cells were used for the ChIRP-MS experiment (100 million - 500 million cells depending on the cell type). Summarily, cell harvesting, lysis, disruption, and ChIRP procedures were performed as previously described(20). Thereafter, protein samples were size-separated on bis-tris SDSPAGE gels (Invitrogen) then subjected to western blot assay and mass spectrometry.

\section{Establishment of a xenograft tumor mouse model}

MHCC97H-Lv-Inc-SCARF1, and HepG2-Lv-Inc-SCARF1 cells were subcutaneously inoculated into the left armpits of 6 male BALB/c nude mice (4-6 weeks old), while control cell lines were inoculated into the right armpit. The sizes of subcutaneous tumors were measured at day $0,7,14,21$, and 28 after injection, And on the $28^{\text {th }}$ day, all animals were euthanized, active tumors removed and cut into small pieces $\left(2 \times 2 \times 2 \mathrm{~mm}^{3}\right)$. Orthotopic implantation was conducted on 24 male athymic BALB/c nude mice (4-6 weeks old). Briefly, each mouse was orthotopically implanted with a tumor mass, into the left lobe of their liver (12 mice per group). 6 mice were implanted with Inc-SCARF1 overexpression tumor mass while the other six were implanted with controls. All mice were euthanized by intraperitoneal injection of pentobarbital, after 4 weeks, then tumor sizes, location, and frequency of both intrahepatic and extrahepatic tumors detected and measured. Athymic BALB/c nude mice were housed in laminar-flow cabinets, under specific pathogenfree conditions, and handled according to the recommendations of the ARRIVE guidelines for the care and use of laboratory animals. All related experimental protocols were approved by the Shanghai Medical Experimental Animal Care Committee. 


\section{Statistical analysis}

All statistical analyses and illustration were performed using SPSS version 23.0 for Windows (SPSS Inc., Chicago, IL, USA) and Prism.v8.0 for Mac (GraphPad Software, La Jolla, CA, USA). Continuous data were first subjected to normality tests, then expressed as means \pm standard errors of the mean (SE). Differences in tumor recurrence, between control and treatment groups, were determined using the Chi-square test, while cumulative survival and recurrence rates were estimated using the Kaplan-Meier method with a logrank test. Independent prognostic factors were calculated using the Cox proportional hazards regression model. Data followed by $P<0.05$ were considered statistically significant.

\section{Results}

\section{Differential IncRNAs expression and aberrant methylation profiles in HCC tissues}

Microarray analysis of IncRNA expression profiles in eight pairs of HCC and adjacent nontumor tissues revealed 1413 up-regulated and 1242 down-regulated IncRNAs (fold-change $\geq 2, P$ value $<0.05$, at least 12 of 16 samples had flags in present or marginal). Profiles of the differentially expressed IncRNAs, after standardization, are shown in Figure 1A-B. Next, we applied methylated DNA immunoprecipitation (MeDIP) and Arraystar Human LncRNA Promotor Microarray analyses to screen differentially methylated IncRNAs and identify those with aberrant methylation during occurrence and development of HCC. Analysis of differentially methylated regions (DMRs) in the methylation microarray profiles, according to AMS result (Probes in DMR $\geq 2$, ABS (AMS_dif) $\geq 8, P$ value $<0.05$ ), revealed a total of 1487 AMS DMRs within the promoters of 1737 genes. A summary of IncRNAs with statistically significant differences in promoter methylation levels is shown in Supplementary Table 3. To select hypermethylated and down-regulated IncRNAs in HCC, we combined and analyzed data from two microarray profiles. Finally, a total of 22 downregulated IncRNAs, with hypermethylation of the promoter region, were selected as candidates for further validation (Table 1 and Figure 1C). 
Table 1

Candidate IncRNAs scanned by conjoint analysis of IncRNA expression microarray and IncRNA promoter microarray

\section{Methylation}

N T

16.178

$17.1222 \quad 26.9178$

$17.3314-28.3864$

17.3314

16.0553

16.9914

16.9914

28.3864

26.0003

9.9450

0.0052

AK056817

9.0257

0.0075 SRD5A1P1

0.0075 RP1-

93C23.1

$\begin{array}{lllll}10.7896 & 19.6320 & 8.8424 & 0.0143 & \text { HTR7P1 }\end{array}$

$12.1433 \quad 21.9067$

9.7634

0.0162 AK091100

9.2029

0.0180

RP11-

755B 10.3

17.7543

27.1246

9.3703

0.0189

AK127534

13.3124

0.0201

AC139666.1

9.1130

0.0210 MTND4P25

0.0212 MT1XP1

8.3851

12.3727

0.0222

CTB-3601.7

12.3727

0.0222

CTB-3601.6

10.1531

0.0225

AC115617.2

0.0284 AY216265

9.3858

(.0291

0.029

LOC113230

0.0302 RP5-

1154E9.6

$\begin{array}{lll}8.9659 & 0.0302 & \text { RP5- } \\ & & 1154 \mathrm{E} 9.6\end{array}$

$\begin{array}{lllll}13.9922 & 25.8375 & 11.8454 & 0.0373 & \text { MT-ATP8 }\end{array}$

0.0386

AC013437.2

$10.4596 \quad 18.7903$

8.3307

0.0429

11.952

\section{Expression}

$\begin{array}{lll}\mathrm{p}- & \mathrm{FC} & \mathrm{T}\end{array}$

$\begin{array}{llll}0.0172 & 2.0000 & 4.5590 & 5.5590\end{array}$

$\begin{array}{llll}0.0066 & 3.6341 & 4.7181 & 6.5797\end{array}$

$\begin{array}{llll}0.0168 & 3.1465 & 10.2668 & 11.9205\end{array}$

$0.0026 \quad 2.2142$

$8.7211 \quad 9.8679$

0.0026

3.0753

9.1655

10.7862

$0.0125 \quad 3.3732$

6.9961

8.7502

0.0094

2.4237

6.3543

7.6315

$\begin{array}{llll}0.0398 & 2.2662 & 7.4462 & 8.6265\end{array}$

0.0032

3.6912

7.6804

9.5645

0.0237

3.0180

5.3224

6.9160

$\begin{array}{llll}0.0212 & 3.4454 & 5.1438 & 6.9285\end{array}$

0.0068

2.3892

5.4296

6.6861

$\begin{array}{llll}0.0099 & 10.6864 & 13.3389 & 16.7566\end{array}$

$21.2992 \quad 9.3467$

$\begin{array}{lll}\text { AC009960.8 } & 0.0092 & 3.3304\end{array}$

$0.0371 \quad 2.5068$

$14.7387 \quad 16.2321$

$15.1197 \quad 16.7862$

$10.7070 \quad 11.9153$

$10.8776 \quad 12.2400$

$\begin{array}{llll}0.0365 & 2.5710 & 10.8776 & 12.2400 \\ 0.0128 & 2.8201 & 11.9266 & 13.4224\end{array}$

$\begin{array}{llll}0.0229 & 2.2815 & 7.7764 & 8.9664\end{array}$

0.0429




\section{Profiles of expression and methylation of candidate IncRNAs in HCC tissues and cell lines}

To verify aberrantly expressed IncRNAs, we performed qRT-PCR to determine their levels of expression in 8 pairs of HCC alongside peritumoral tissues. Results showed that 20 candidate IncRNAs were significantly downregulated in HCC, but there was no significant difference in expression of SRD5A1P1 and RP1-93C3.1, between $\mathrm{HCC}$ and paired nontumor tissues (Figure 2A). Next, we validated expression of candidate IncRNAs in another 52 pairs of HCC tissues, and found that they were significantly down- regulated in HCC relative to nontumor tissues (Figure 2B). Further analysis of IncRNA promoters revealed presence of CpG islands in 13 candidates, namely SCARF1, AK126915, MST1P9, AK056817, SRD5A1P1, RP1-93C23.1, HTR7P1, RP11-755B10.3, AK127534, CTB-3601.7, CTB-3601.6, AY216265, LOC113230. CTB-3601.6 and CTB-3601.7. On the other hand, SRD5A1P1 and RP1-93C sequences originated from the same genomic locus and shared the identical promoter sequences. Furthermore, we combined methylation specific PCR (MSP) and bisulfite genomic sequencing (BSP) analyses, in the tissues from which the IncRNA microarray data were obtained, to detect presence of $\mathrm{CpG}$ island methylation in the candidates MSP results revealed that 4 out of 13 IncRNAs, namely SCARF1a, AK126915, MST1P9 and AK056817, were significantly promoter hypermethylated in HCC tissue samples (Figure 2D, Table 2). Similarly, BSP results confirmed a remarkable hypermethylation of promoters of 4 candidate IncRNAs in HCC tissues (Figure 2C, Table 3). Furthermore, we found statistically significant differences in the promoter methylation status between 30 pairs of HCC and peritumor tissues(Supplementary Table 4). Notably, Lnc-SCARF1 exhibited significant differences in promoter methylation between $\mathrm{HCC}$ and peritumor tissues $(P<0.001)$, and was ultimately was chosen as a candidate for further explorations.

Table 2

BSP result of 8 samples

\begin{tabular}{|llll|}
\hline LncRNA & Methylation in HCC (\%) & Methylation in Peritumor (\%) & P value \\
\hline SCARF1 & $64.8 \pm 20.4$ & $26.6 \pm 5.3$ & $<0.001$ \\
\hline AK126915 & $74.3 \pm 9.2$ & $38.2 \pm 19.8$ & 0.0013 \\
\hline MST1P9 & $59.2 \pm 21.6$ & $37.5 \pm 17.4$ & 0.043 \\
\hline AK056817 & $60.7 \pm 21.0$ & $36.1 \pm 20.6$ & 0.033 \\
\hline
\end{tabular}


Table 3

MSP result of 8 samples

\begin{tabular}{|lll|}
\hline LncRNA & Methylation in HCC (M/U) & Methylation in Peritumor $(M / U)$ \\
\hline SCARF1 & $8 / 0$ & $1 / 7$ \\
AK126915 & $6 / 2$ & $2 / 6$ \\
\hline MST1P9 & $7 / 1$ & $2 / 6$ \\
\hline AK056817 & $5 / 3$ & $2 / 6$ \\
\hline
\end{tabular}

Table 4

MSP result of 30 samples

\begin{tabular}{|lll|}
\hline LncRNA & Methylation in HCC (M/U) & Methylation in Peritumor (M/U) \\
\hline SCARF1 & $23 / 7$ & $8 / 22$ \\
\hline AK126915 & $19 / 11$ & $6 / 24$ \\
\hline MST1P9 & $24 / 6$ & $12 / 18$ \\
\hline AK056817 & $20 / 10$ & $9 / 21$ \\
\hline
\end{tabular}

\section{Hypermethylation in the promoter of Inc-SCARF1 induces its downregulation in HCC tissues}

Both MSP and BSP results revealed hypermethylation of Inc-SCARF1 promoter in HCC relative to adjacent normal tissues, indicating that most of the $\mathrm{CpG}$ sites within the $\mathrm{CpG}$ islands of the promoter were hypermethylated in HCC tissues (Figure 2C). Furthermore, Inc-SCARF1 was significantly overexpressed in the normal liver cell line LO2 relative to HCC cell lines (Figure 3A). Next, we used the inhibitor of DNA methylation, 5-aza-deoxycytidine (5-AZA), to investigate the relationship between hypermethylation in promoter and downregulation of Inc-SCARF1. Two types of HCC cell lines, namely HepG2 and MHCC-97H, which earlier exhibited downregulation of Inc-SCARF1, were chosen and cultured with two different concentrations of 5-AZA (30, and $60 \mu \mathrm{M})$. After treatment, we observed upregulation of Inc-SCARF1 expression in HepG2 and MHCC-97H cells, in a concentration-dependent manner (Figure 3B). These results indicated that hypermethylation in the promoter of Inc-SCARF1 induces its downregulation in HCC.

\section{Over-expression of Inc-SCARF1 inhibits tumor proliferation and migration of HCC in vitro}


To investigate Inc-SCARF1's biological function, we successfully constructed lentiviral vectors harboring Inc-SCARF1 and stably transfected HepG2 and MHCC-97H cell lines (Figure 3C). Next, we tested the effect of overexpressing Inc-SCARF1 on proliferation, migration and apoptosis of two transfected cell lines, alongside invalid controls, using a series of assays, and found that its overexpression significantly suppresses proliferation of HepG2 and MHCC-97H cells (Figure 3D). Moreover, we characterized the effects of Inc-SCARF1 overexpression on migration, and as expected, overexpressing Inc-SCARF1 inhibited cell migration and suppressed the number of migrating cells, with upregulation of Inc-SCARF1 was significantly lower than that of control cells (Figure 3E and F). Furthermore, flow cytometry results confirmed that enforced expression of Inc-SCARF1 induced apoptosis of HepG2 and MHCC-97H cells (Figure 3G).

\section{Lnc-SCARF1 suppresses HCC progression, intrahepatic and pulmonary metastases in vivo}

To explore the effect of Lnc-SCARF1 in carcinogenesis in vivo, Lnc-SCARF1 upregulated and control HepG2 and MHCC-97H cell lines tagged with GFP were subcutaneously implanted and orthotopically transplanted into mice. Tumor sizes of MHCC-97H-LV-SCARF1 and HepG2-LV-SCARF1 xenografts were $0.037 \pm 0.070$ and $0.102 \pm 0.073 \mathrm{~cm}^{3}$, respectively, which were significantly smaller than those obtained from MHCC-97HMock $\left(0.164 \pm 0.108 \mathrm{~cm}^{3}\right)$ and HepG2-Mock $\left(0.429 \pm 0.349 \mathrm{~cm}^{3}, P<0.05\right)$. The results corroborated findings in the orthotropic transplantation cohort $\left(0.037 \pm 0.033 \mathrm{~cm}^{3}\right.$ and $0.006 \pm 0.008 \mathrm{~cm}^{3}$ versus $1.005 \pm$ $0.913 \mathrm{~cm}^{3}$ and $0.208 \pm 0.216 \mathrm{~cm}^{3}$, respectively, $P<0.05$ ) (Figure 4A, B). Next, we investigated the effect of Lnc-SCARF1 on HCC metastasis in vivo, and found that both intrahepatic and pulmonary metastases were significantly suppressed in mice treated with LV-SCARF1 transfectants. Notably, the number of intrahepatic metastatic nodules of each grade were significantly higher in MHCC97H-Mock and HepG2-Mock mice, relative to their counterparts in the MHCC-97H-LV-SCARF1 and HepG2-LV-SCARF1 groups (Figure 4C). In addition, 83.3\% (5/6) of MHCC-97H-Mock mice exhibited pulmonary metastasis (Figure 4D), which was significantly higher than that observed in the MHCC-97H -LV-SCARF1 group (0/6).

\section{Lnc-SCARF1 interacts with functional domains of signaling proteins, to act as a class of CUL9 modulators and suppress HCC metastasis}

Next, we applied chromatin isolation by RNA purification (ChIRP) to determine whether Inc-SCARF1 plays a role in inhibiting tumor growth and metastasis by binding to specific proteins. Results from mass spectrometry (MS) analysis revealed that CUL9 specifically bound to Inc-SCARF1 (Figure 4E). To further validate the interaction between Inc-SCARF1 and CUL9, we performed western blot assay to detect CUL9 in both HepG2 and MHCC-97H cell lines. Results revealed a marked upregulation of CUL9 protein in these HCC cell lines after LV-SCARF1 transfection (Figure 4F). 


\section{Discussion}

Hepatocellular carcinoma is a lethal disease characterized by high malignancy and an overall five-year survival rate of $5-30 \%(21)$. Accumulating evidences have shown that IncRNAs play a vital role in carcinogenesis of HCC, while aberrant DNA methylation regulates cellular biological function of liver cancer(22). Moreover, previous studies have applied genome-wide DNA methylation analysis to reveal several epigenetically dysregulated IncRNAs in human renal cancer(16) and breast cancer(23). However, only a handful of researches have focused on the role of demethylation-mediated IncRNAs dysregulation in occurrence and development of HCC. In the present study, we investigated the function of an HCC-specific IncRNA-SCARF1, and found that hypermethylation of promoter CpGls could silence its expression, and subsequently promote progression and metastasis of hepatocellular carcinoma via binding to CUL9's target gene .

Numerous studies have demonstrated the essential regulatory role of IncRNAs in tumorigenesis, recurrence and metastasis(24). Additional evidences have revealed that several carcinogenic IncRNAs, such as IncHULC(25), HEIH(26) and MVIH(27), are not only overexpressed in but are also closely associated with prognosis of HCC patients. Meanwhile, several IncRNAs have been shown to act as tumor suppressors. For example, MEG3 which is downregulated in HCC tissues, was regulated by miR-29a and induced cell apoptosis of $\mathrm{HCC}(28)$. Results of the present study indicated that Inc-SCARF1 expression significantly suppressed tumor progression both in vitro and in vivo. Specifically, Inc-SCARF1 up-regulation suppressed cell proliferation, migration and apoptosis of HCC cell lines, while results from both subcutaneous and orthotropic transplantation models showed that Inc-SCARF1 significantly suppressed intrahepatic and pulmonary metastasis of HCC.

DNA hypermethylation modification of CpGls within the promoter of tumor suppression genes (TSGs) has been found to contribute to transcriptional silencing and HCC carcinogenesis(29). These TSGs, including RASSF1A, p16, and APC, have also been shown to play a key role in regulation of cell apoptosis, cell cycle and other tumor-related signaling(30). Results of the present study revealed that Inc-SCARF1 was downregulated in HCC, but the frequency of promoter methylation was high. To ascertain whether inactivation of Inc-SCARF1 was related to hypermethylation of its promoter, we used 5-AZA, an inhibitor of methylation, to prevent promoter hypermethylation in vitro. Results showed that lack or weak expression of Inc-SCARF1 in HCC tissues was closely associated with its hypermethylated promoter CpGls, consistent with the findings of a previous study that showed that methyltransferase EZH2 regulated the levels of H3K27me3 at promoters of IncRNAs thereby regulating their expression(31). This result further indicated that IncRNAs regulate promoter methylation in a similar fashion to that of protein coding genes. Moreover, another study found that an inverse correlation between linc-POU3F3 and POU3F3 gene was associated with regulation of EZH2(32), suggesting that IncRNAs might be associated with chromatin modifying complexes where they modulate their neighboring gene transcription and expression by regulating the methylation status. However, only a handful of studies have described the underlying mechanism of promoter methylation in modulating expression of IncRNAs in HCC. Consequently, we sought to ascertain 
whether regulation of methyltransferases, such as EZH2, was correlated to the methylation status of IncSCARF1 in HCC.

LncRNAs exert their regulatory role by interacting with transcriptional regulatory factors or chromatin protein complexes. For example, Lnc-HElH was not only associated with EZH2 but competitively recruited it to specific genomic DNA regions, and repressed the EZH2 target genes(26). Additional evidences have also demonstrated that IncRNAs are directly correlated with functional domains of signaling proteins and they activate the signaling pathway that regulates tumorigenesis and progression. For instance, Lnc-LET was found to play a role in the stabilization of signaling proteins thereby leading to hypoxia-induced cell invasion of $\mathrm{HCC}(33)$. On the other hand, Lnc-MEG3 reportedly induced expression of p53 by activating the p53 signaling pathway, further inhibiting cell proliferation and inducing cell apoptosis(34). In order to clarify the potential regulating signaling pathway of Inc-SCARF1, we utilized ChIRP to pull down and purify chromatin protein complexes that interact with Inc-SCARF1, then employed mass spectrometry to further screen target proteins that are involved in relevant tumor signaling. MS results revealed that Cullin 9 (CUL9) specifically interacted with Inc-SCARF1. CUL9, which is predominantly localized in the cytoplasm, is a member of the cullin family of E3 ubiquitin ligases(35). Previous studies have shown that deletion of the CUL9 gene resulted in spontaneous tumor development, promoted myc-induced lymphomagenesis, and induced tumorigenesis in mice (36). Results of the present study indicated that overexpression of IncSCARF1 reversed CUL9 downregulation in HCC cell lines, suggesting that Lnc-SCARF1 might induce tumor suppression by regulating CUL9 expression and the corresponding signaling pathway. Previous studies have shown that the function of CUL 9 in tumor inhibition is largely mediated by p53 signaling, with CUL 9 deletion associated with suppressed DNA damage-induced apoptosis(36, 37). Moreover, other evidences have confirmed that CUL9 might promote ubiquitination and degradation of survivin. Notably, products of three $3 \mathrm{M}$ genes, namely CUL7, OBSL 1 , and CCDC8, formed the $3 \mathrm{M}$ complex that modulated CUL 9 and its substrate survivin, which maintains microtubule and genome integrity, while impairment of survivin was associated with carcinogenesis(38).

In summary, we revealed that Inc-SCARF1 plays an important role in HCC suppression. Specifically, promoter methylation directly regulates expression of Inc-SCARF1, which subsequently modulates proliferation, metastasis and apoptosis of HCC cells by interacting with its target gene CUL9. However, the mechanism of Inc-SCARF1's regulation by promoter methylation, and the interaction between SCARF1 with its target gene CUL9, remain unclear. Elucidating the mechanism through which promoter methylation regulates IncRNAs, and the potential interaction between SCARF1 and CUL9 are imperative to understanding the mechanism underlying the role of IncRNAs in HCC development and progression, and providing new therapeutic signatures and targets.

\section{Conclusion}

Taken together, our findings indicate that LnCRNA-SCARF1 plays important roles in HCC progression by interacting with CUL9, thus may serve as a prognostic biomarker or an effective target for future development of HCC therapies. 


\section{Abbreviations}

HCC, Hepatocellular Carcinoma; LncRNA, Long noncoding RNA; NASH, Non-alcoholic Steatohepatitis; MeDIP, Methylated DNA Immunoprecipitation; AMS, Absolute Methylation Score; DMR, Differentially Methylated Region; qRT-PCR, Quantitative Real-time Polymerase Chain Reaction; BSP, Bisulphite Modification Combining Sequencing PCR; MSP, Methylation Specific PCR; DMEM, Dulbecco's Modified Eagle Medium; ChIRP, Chromatin Isolation by RNA Purification; MS, Mass Spectrometry

\section{Declarations}

\section{Ethics approval and consent to participate:}

All research had been performed in accordance with the Declaration of Helsinki. All methods related to animal experiments are in accordance with ARRIVE guidelines. All related experimental protocols were approved by the Shanghai Medical Experimental Animal Care Committee. The present study was approved by the Ethics Committee of Zhongshan Hospital, Fudan University (approval no.Y2013-033), and informed consent was provided by all patients.

\section{Consent for publication:}

Not applicable.

\section{Availability of data and materials:}

The datasets used and/or analyzed during the current study are available from the corresponding author on reasonable request.

\section{Competing interests:}

The authors declare that they have no competing interests.

\section{Funding:}

This work was supported by the National Natural Science Foundation of China (No. 82173261) and Natural Science Foundation of Shanghai(20ZR1411400).

\section{Author Contributions:}

Boyi Liao, Peiran Huang and Xiangyu Zhang performed all experiments; Xinyu Wang and Kaiqian Zhou drafted the manuscript; Feiyu Chen and Cheng Zhou interpreted the results; Lei Yu and Jie Hu performed 
statistical analyses.;Jian Zhou critically revised the manuscript for important intellectual content. Jie $\mathrm{Hu}$ and Zheng Wang conceived and designed the study. All authors read and approved the final manuscript.

\section{Acknowledgements:}

Not applicable.

\section{References}

1. Bray F, Ferlay J, Soerjomataram I, Siegel RL, Torre LA, Jemal A. Global cancer statistics 2018: GLOBOCAN estimates of incidence and mortality worldwide for 36 cancers in 185 countries. CA Cancer J Clin. 2018;68(6):394-424.

2. Hartke J, Johnson M, Ghabril M. The diagnosis and treatment of hepatocellular carcinoma. Semin Diagn Pathol. 2017;34(2):153-9.

3. Waghray A, Murali AR, Menon KN. Hepatocellular carcinoma: From diagnosis to treatment. World J Hepatol. 2015;7(8):1020-9.

4. Mittal S, Sada YH, El-Serag HB, Kanwal F, Duan Z, Temple S, et al. Temporal Trends of Nonalcoholic Fatty Liver Disease-Related Hepatocellular Carcinoma in the Veteran Affairs Population. Clin Gastroenterol H. 2015;13(3):594-601.

5. Li L, Jin R, Zhang X, Lv F, Liu L, Liu D, et al. Oncogenic activation of glypican-3 by c-Myc in human hepatocellular carcinoma. Hepatology. 2012;56(4):1380-90.

6. Tullo A, Sbisa E. Mutation of p53 Tumor Suppressor Gene in Hepatocellular Carcinoma. Methods Mol Med. 2000;45:113-30.

7. Consortium EP. An integrated encyclopedia of DNA elements in the human genome. Nature. 2012;489(7414):57-74.

8. Guttman M, Amit I, Garber M, French C, Lin MF, Feldser D, et al. Chromatin signature reveals over a thousand highly conserved large non-coding RNAs in mammals. Nature. 2009;458(7235):223-7.

9. Lai F, Shiekhattar R. Where long noncoding RNAs meet DNA methylation. Cell research. 2014;24(3):263-4.

10. Mattick JS. The genetic signatures of noncoding RNAs. PLoS genetics. 2009;5(4):e1000459.

11. Ramnarine VR, Kobelev M, Gibb EA, Nouri M, Lin D, Wang Y, et al. The evolution of long noncoding RNA acceptance in prostate cancer initiation, progression, and its clinical utility in disease management. Eur Urol. 2019.

12. Zheng YJ, Zhao JY, Liang TS, Wang P, Wang J, Yang DK, et al. Long noncoding RNA SMAD5-AS1 acts as a microRNA-106a-5p sponge to promote epithelial mesenchymal transition in nasopharyngeal carcinoma. FASEB J. 2019:fj201900803R. 
13. Yang X, Sun L, Wang L, Yao B, Mo H, Yang W. LncRNA SNHG7 accelerates the proliferation, migration and invasion of hepatocellular carcinoma cells via regulating miR-122-5p and RPL4. Biomed Pharmacother. 2019;118:109386.

14. Pan W, Li W, Zhao J, Huang Z, Zhao J, Chen S, et al. IncRNA-PDPK2P promotes hepatocellular carcinoma progression through the PDK1/AKT/Caspase 3 pathway. Mol Oncol. 2019;13(10):2246-58.

15. Cai C, Xie X, Zhou J, Fang X, Wang F, Wang M. Identification of TAF1, SAT1, and ARHGEF9 as DNA methylation biomarkers for hepatocellular carcinoma. J Cell Physiol. 2019.

16. Zhou H, Guo L, Yao W, Shi R, Yu G, Xu H, et al. Silencing of tumor-suppressive NR_023387 in renal cell carcinoma via promoter hypermethylation and HNF4A deficiency. J Cell Physiol. 2019.

17. Villanueva A, Portela A, Sayols S, Battiston C, Hoshida Y, Mendez-Gonzalez J, et al. DNA methylationbased prognosis and epidrivers in hepatocellular carcinoma. Hepatology. 2015;61(6):1945-56.

18. Heller G, Babinsky VN, Ziegler B, Weinzierl M, Noll C, Altenberger C, et al. Genome-wide CpG island methylation analyses in non-small cell lung cancer patients. Carcinogenesis. 2013;34(3):513-21.

19. Luo X, Hong L, Cheng C, Li N, Zhao X, Shi F, et al. DNMT1 mediates metabolic reprogramming induced by Epstein-Barr virus latent membrane protein 1 and reversed by grifolin in nasopharyngeal carcinoma. Cell Death Dis. 2018;9(6):619.

20. Chu C, Qu K, Zhong FL, Artandi SE, Chang HY. Genomic maps of Iong noncoding RNA occupancy reveal principles of RNA-chromatin interactions. Mol Cell. 2011;44(4):667-78.

21. Allemani C, Matsuda T, Di Carlo V, Harewood R, Matz M, Niksic M, et al. Global surveillance of trends in cancer survival 2000-14 (CONCORD-3): analysis of individual records for 37513025 patients diagnosed with one of 18 cancers from 322 population-based registries in 71 countries. Lancet. 2018;391(10125):1023-75.

22. Lim LJ, Wong SYS, Huang F, Lim S, Chong SS, Ooi LL, et al. Roles and Regulation of Long Noncoding RNAs in Hepatocellular Carcinoma. Cancer Res. 2019.

23. Li Y, Zhang Y, Li S, Lu J, Chen J, Wang Y, et al. Genome-wide DNA methylome analysis reveals epigenetically dysregulated non-coding RNAs in human breast cancer. Sci Rep. 2015;5:8790.

24. Bach DH, Leek SK. Long noncoding RNAs in cancer cells. Cancer Letters. 2018;419:152-66.

25. Hammerle M, Gutschner T, Uckelmann H, Ozgur S, Fiskin E, Gross M, et al. Posttranscriptional destabilization of the liver-specific long noncoding RNA HULC by the IGF2 mRNA-binding protein 1 (IGF2BP1). Hepatology. 2013;58(5):1703-12.

26. Yang F, Zhang L, Huo XS, Yuan JH, Xu D, Yuan SX, et al. Long noncoding RNA high expression in hepatocellular carcinoma facilitates tumor growth through enhancer of zeste homolog 2 in humans. Hepatology. 2011;54(5):1679-89.

27. Yuan SX, Yang F, Yang Y, Tao QF, Zhang J, Huang G, et al. Long noncoding RNA associated with microvascular invasion in hepatocellular carcinoma promotes angiogenesis and serves as a predictor for hepatocellular carcinoma patients' poor recurrence-free survival after hepatectomy. Hepatology. 2012;56(6):2231-41. 
28. Braconi C, Kogure T, Valeri N, Huang N, Nuovo G, Costinean S, et al. microRNA-29 can regulate expression of the long non-coding RNA gene MEG3 in hepatocellular cancer. Oncogene. 2011;30(47):4750-6.

29. Yuan SX, Zhang J, Xu QG, Yang Y, Zhou WP. Long noncoding RNA, the methylation of genomic elements and their emerging crosstalk in hepatocellular carcinoma. Cancer Lett. 2016;379(2):239-44.

30. Timoshkina NN, Kit OI, Vodolazhskiy DI, Soldatkina NV, Gevorkyan YA, Samoylenko NS, et al. Changes in $\mathrm{CpG}$ methylation of APC, CDH13, MLH1, MGMT, P16 and RASSF1A in gastric adenocarcinoma. Annals of Oncology. 2018;29.

31. Wu SC, Kallin EM, Zhang Y. Role of H3K27 methylation in the regulation of IncRNA expression. Cell Res. 2010;20(10):1109-16.

32. Li W, Zheng J, Deng J, You Y, Wu H, Li N, et al. Increased Levels of the Long Intergenic Non-protein Coding RNA POU3F3 Promote DNA Methylation in Esophageal Squamous Cell Carcinoma Cells. Gastroenterology. 2014.

33. Yang F, Huo XS, Yuan SX, Zhang L, Zhou WP, Wang F, et al. Repression of the long noncoding RNA-LET by histone deacetylase 3 contributes to hypoxia-mediated metastasis. Molecular cell. 2013;49(6):1083-96.

34. Benetatos L, Vartholomatos G, Hatzimichael E. MEG3 imprinted gene contribution in tumorigenesis. International journal of cancer Journal international du cancer. 2011;129(4):773-9.

35. Gama V, Swahari V, Schafer J, Kole AJ, Evans A, Huang Y, et al. The E3 ligase PARC mediates the degradation of cytosolic cytochrome c to promote survival in neurons and cancer cells. Science signaling. 2014;7(334):ra67.

36. Pei XH, Bai F, Li Z, Smith MD, Whitewolf G, Jin R, et al. Cytoplasmic CUL9/PARC ubiquitin ligase is a tumor suppressor and promotes p53-dependent apoptosis. Cancer research. 2011;71(8):2969-77.

37. Li Z, Xiong Y. Cytoplasmic E3 ubiquitin ligase CUL9 controls cell proliferation, senescence, apoptosis and genome integrity through p53. Oncogene. 2017.

38. Li Z, Pei XH, Yan J, Yan F, Cappell KM, Whitehurst AW, et al. CUL9 mediates the functions of the 3M complex and ubiquitylates survivin to maintain genome integrity. Molecular cell. 2014;54(5):805-19.

\section{Figures}


A

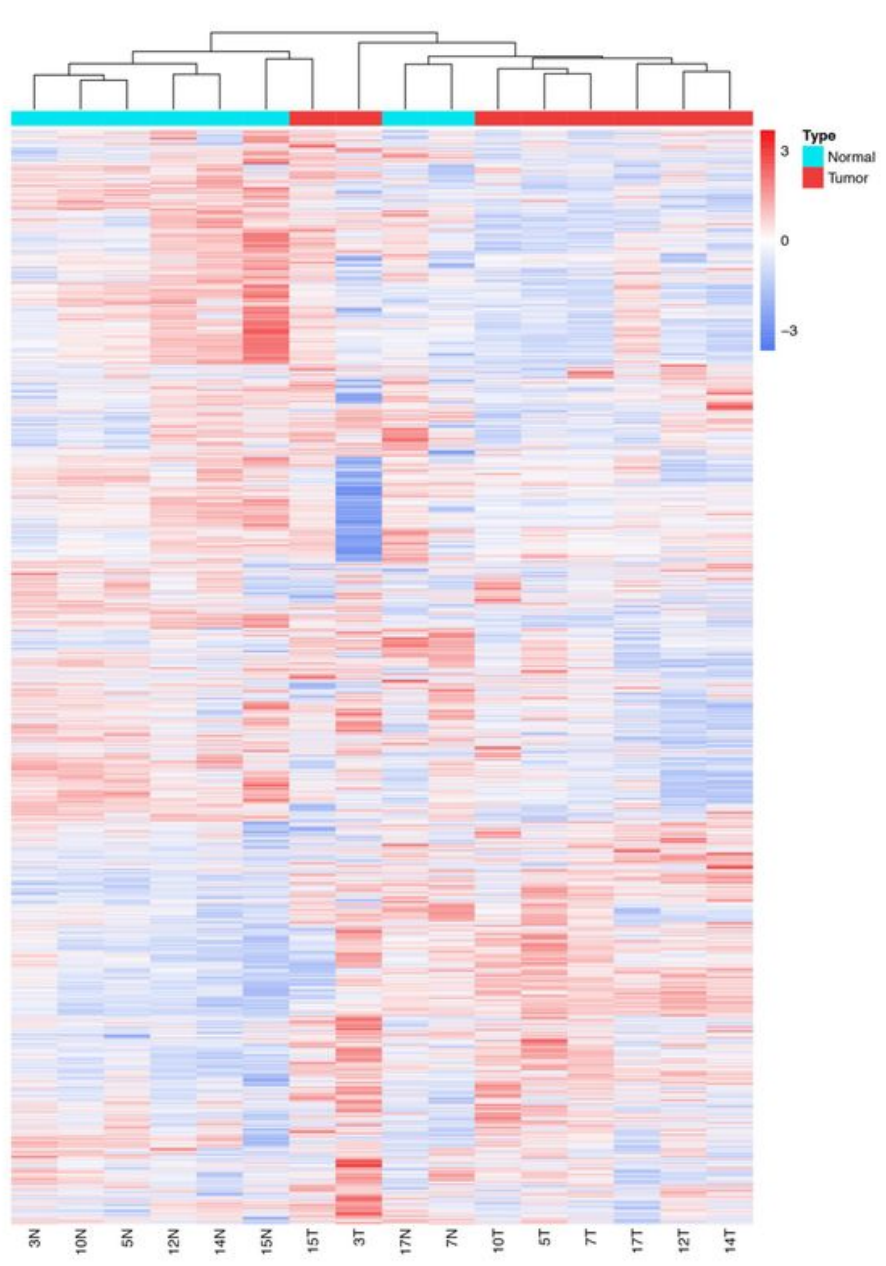

B

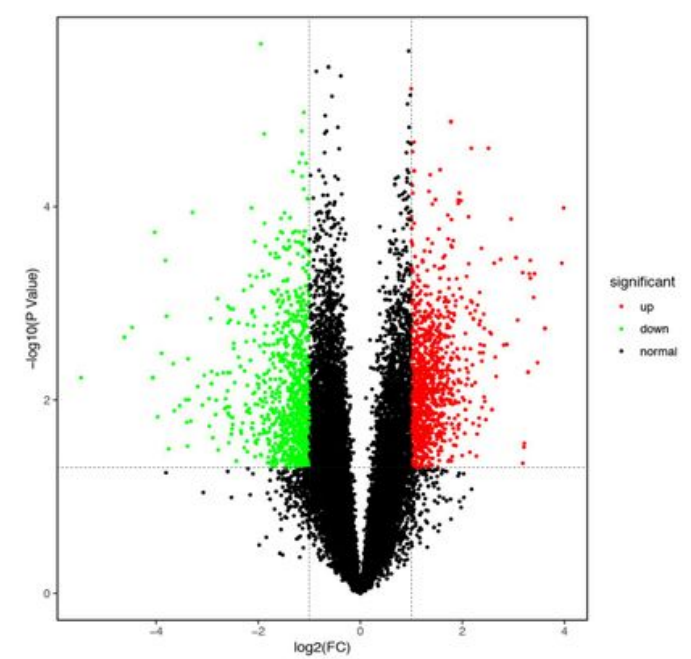

C

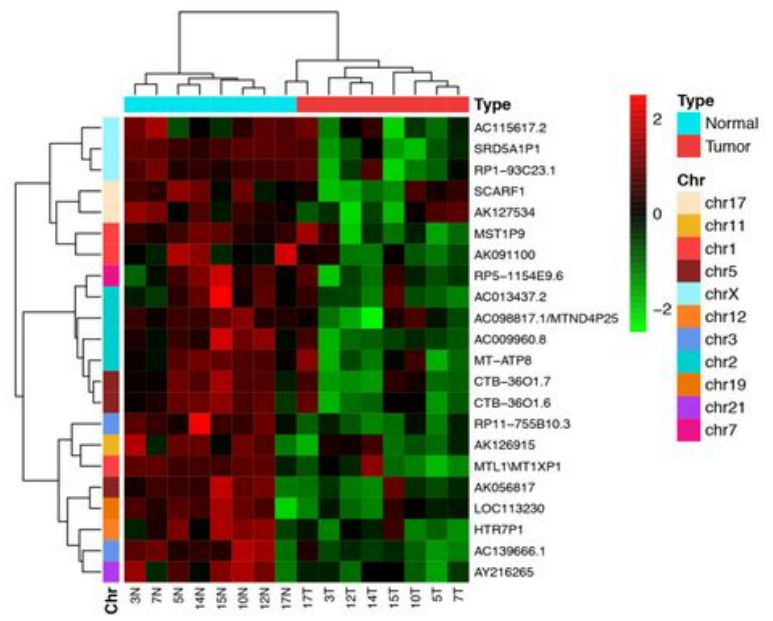

Figure 1

Differential IncRNA expression and aberrant methylation of promoters.

A-B. Heatmap and volcano plot showing differentially expressed IncRNAs between tumor and peritumor tissues. C. Conjoint-based cluster analysis of IncRNA expression microarray and IncRNA promoter microarray. A total of 22 down-regulated IncRNAs with promoter region hypermethylation were selected as candidates. 
A

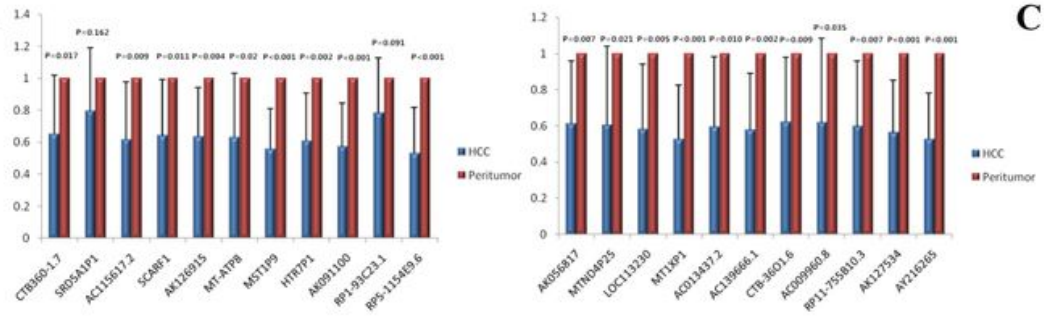

B

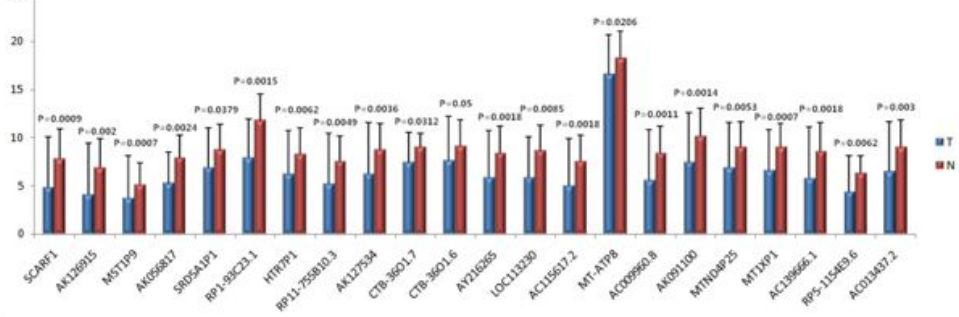

D

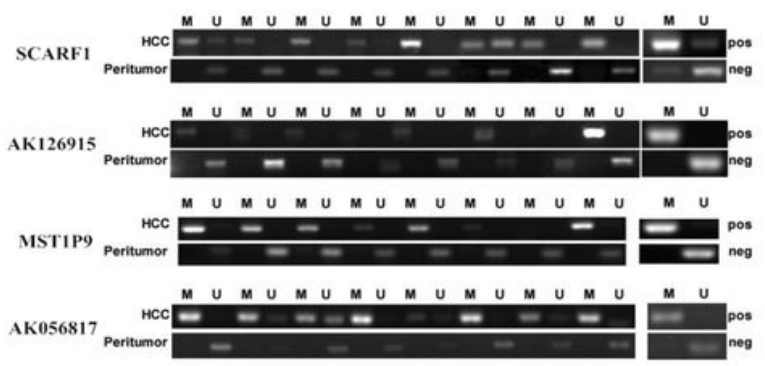

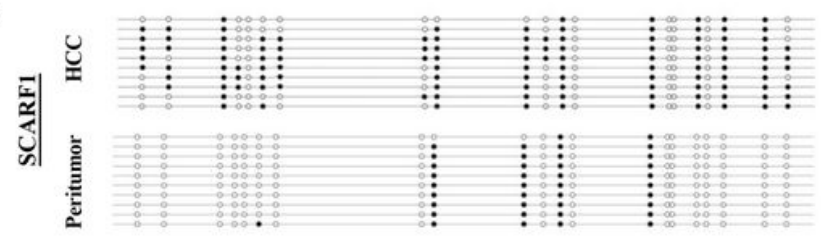
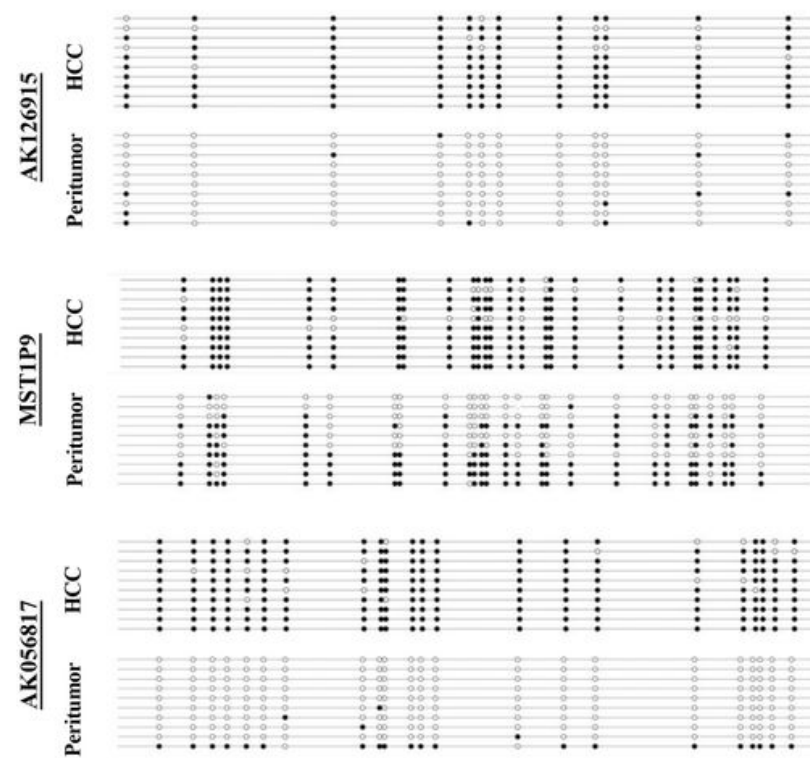

Figure 2

Expression profiles and methylation verification of candidate IncRNAs in HCC tissues and cell lines.

A. qRT-PCR results showing relative expression levels of candidate IncRNAs in HCC and peritumor tissues.

B. Validation of 20 candidate IncRNAs in 52 pairs of HCC tissues downregulated in HCC tissues. C. BSP results showing a remarkable hypermethylated status in the promoter of 4 candidate IncRNAs in 8 HCC tissues. D. Distribution patterns of electrophoretic bands showing marked differences between HCC and peritumor tissues. 
A

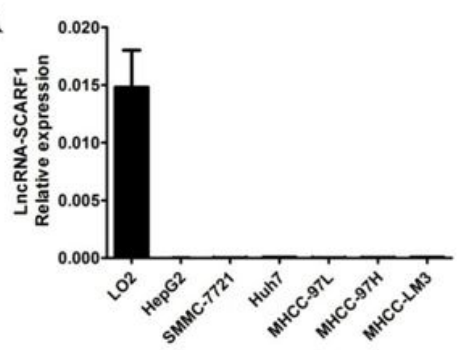

B

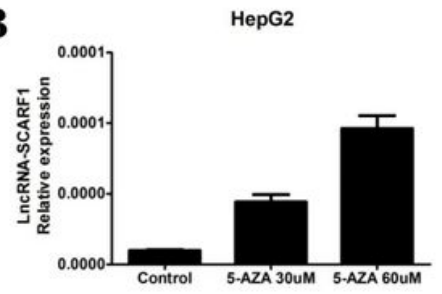

C

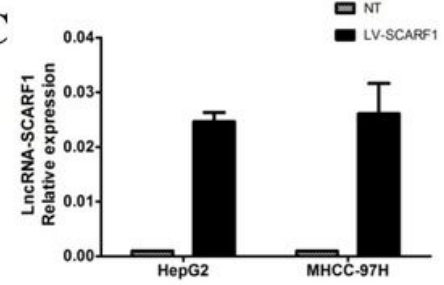

D

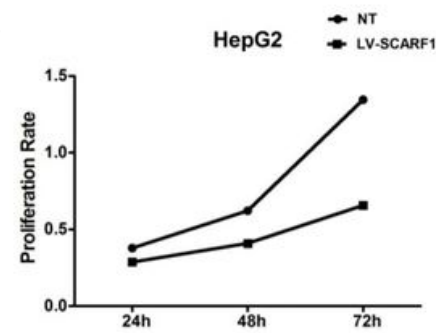

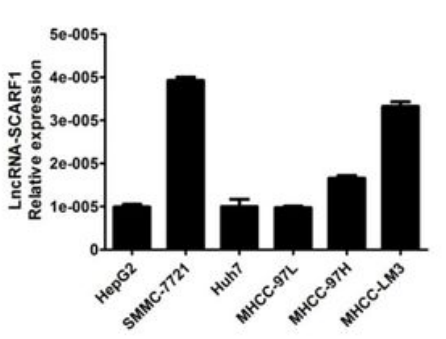

MHCC-97H

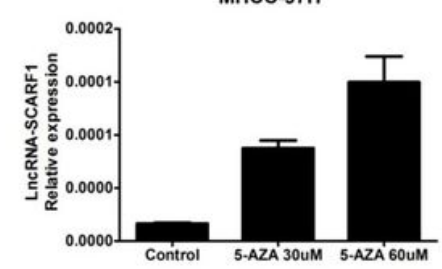

E
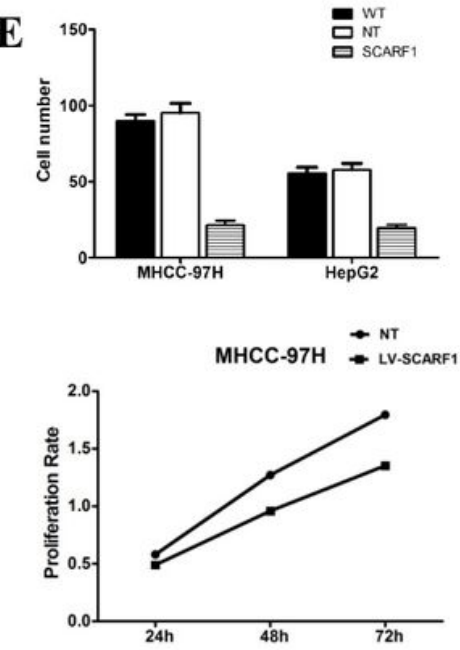

F

FT LV-NT LV-SCARF1

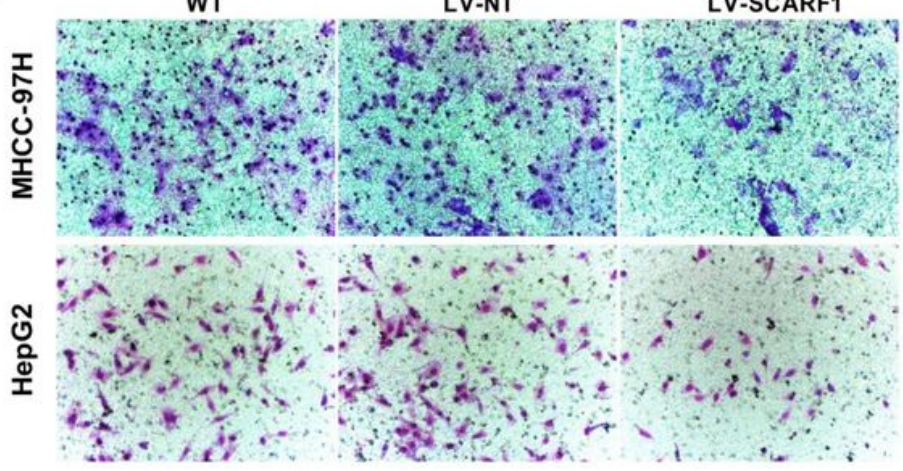

G
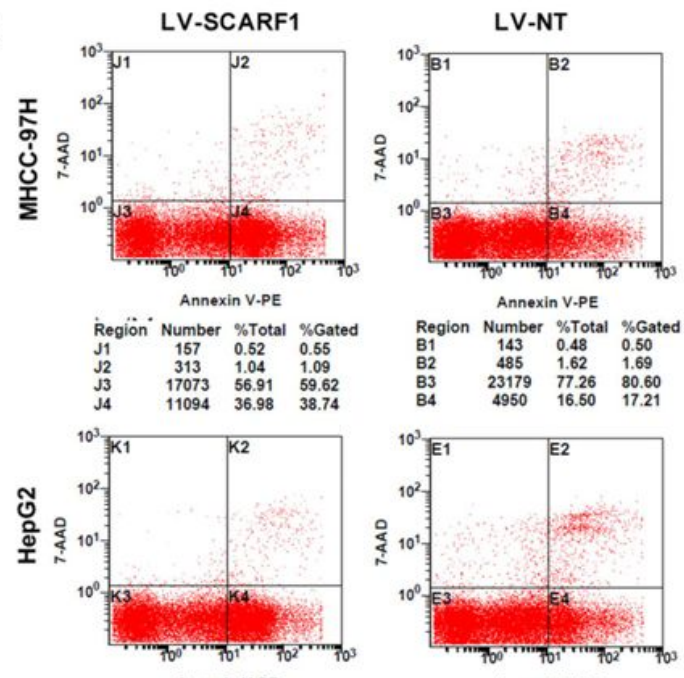

Annexin V.PE

Region Number \%Total \%Gated $\begin{array}{lclll}\text { Region } & \text { Number } & \text { \% Total } & \text { \%Gated } \\ \text { K1 } & 122 & 0.41 & 0.43 \\ \text { K2 } & 306 & 1.02 & 1.08 \\ \text { K3 } & 16784 & 55.95 & 59.41\end{array}$

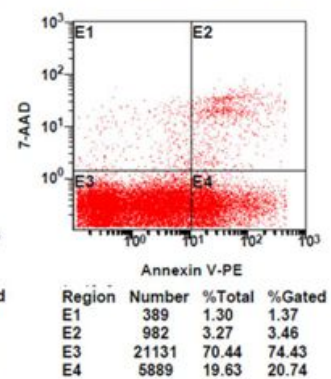

Figure 3

\section{5-AZA upregulated Inc-SCARF1 expression, and inhibited tumor proliferation and migration of HCC in vitro.}

A. Relative expression levels of Inc-SCARF1 in normal liver cell line LO2 and 6 other HCC cell lines. B. Expression level of Inc-SCARF1 in HepG2 and MHCC-97H cells treated with or without 5-AZA. C. Levels of Inc-SCARF1 expression before and after transfection with lentiviral constructs harboring Inc-SCARF1. D. Proliferation curves of 2 HCC cell lines overexpressing Inc-SCARF1 relative to controls. E. Cell numbers (across five microscopic fields at $\times 200$ magnification), of 3 diverse groups. F. Invasive cells localized on the lower surface of Transwell chamber stained with Giemsa. G. Flow cytometry results showing the ratio of apoptotic cells. 
A

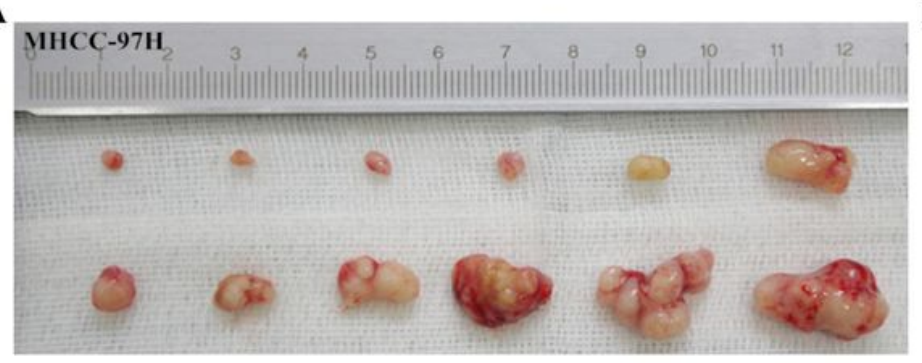

C

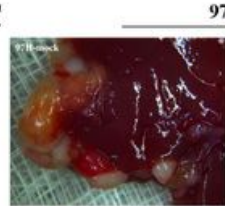

97H
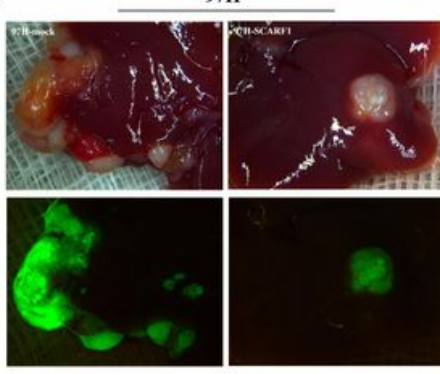

D

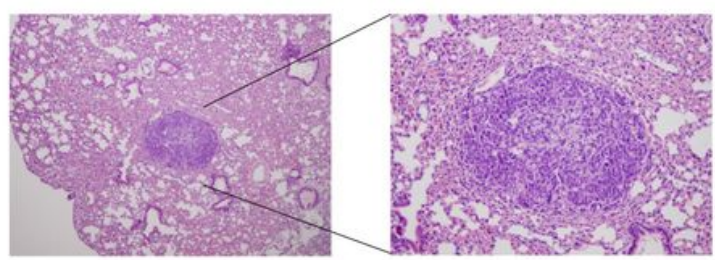

B

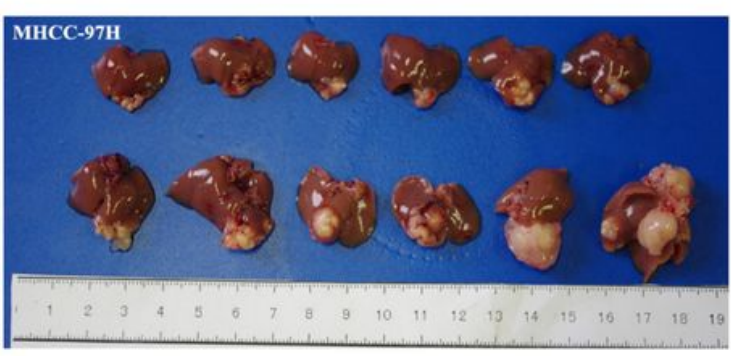

$\mathbf{E}$

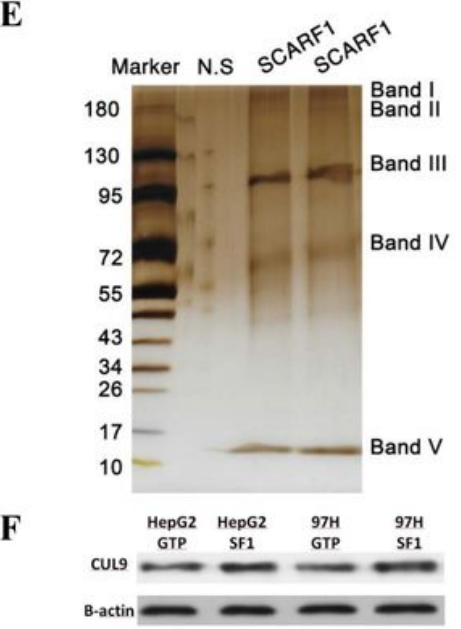

\section{Figure 4}

\section{Effect of over-expressing Lnc-SCARF1 in carcinogenesis in vivo.}

A. Tumor sizes of xenografts across two groups. The top panel shows tumors derived from MHCC-97H-LVSCARF1 cells, while the lower one represents tumors derived from MHCC-97H-mock cells. B. Tumor sizes of orthotropic grafts of two groups. C. Intrahepatic metastasis shown by GFP fluorescence. D. H \& E-stained sections showing pulmonary metastasis in MHCC-97H-LV-SCARF1 mice. E. Chromatin Isolation by RNA Purification (ChIRP) and gel electrophoresis results. F. Levels of CUL9 expression in HCC cell lines before and after LV-SCARF1 transfection.

\section{Supplementary Files}

This is a list of supplementary files associated with this preprint. Click to download.

- SupplementaryMaterials.docx 